Abstract P63 Table 1 Diagnostic accuracy of serum and pleural fluid procalcitonin for complicated parapneumonic effusion in sub-groups

\begin{tabular}{|c|c|c|c|c|c|c|c|}
\hline Sub-group & Sample type & $\begin{array}{l}\text { Number } \\
\text { of patients }\end{array}$ & AUC $(95 \% \mathrm{Cl})$ & Sensitivity $(95 \% \mathrm{CI})$ & $\begin{array}{l}\text { Specificity } \\
(95 \% \mathrm{CI})\end{array}$ & PPV (95\% Cl) & NPV $(95 \%$ Cl) \\
\hline \multirow[t]{2}{*}{ Pleural fluid $\mathrm{pH} \leq 7.2$} & Serum & $\mathrm{n}=20$ & $0.81(0.61$ to 1.00$)$ & $91 \%$ (59 to $100 \%$ ) & $67 \%$ (30 to $93 \%$ ) & $77 \%$ (46 to $95 \%$ ) & $86 \%$ (42 to $100 \%$ ) \\
\hline & Pleural fluid & $\mathrm{n}=20$ & $0.60(0.34$ to 0.86$)$ & $82 \%$ (48 to $97 \%$ ) & $56 \%$ (21 to $86 \%)$ & $69 \%$ (39 to $91 \%$ ) & $72 \%$ (29 to $96 \%$ ) \\
\hline \multirow{2}{*}{$\begin{array}{l}\text { Excluding inpatient } \\
\text { stay }>48 \mathrm{~h}\end{array}$} & Serum & $\mathrm{n}=98$ & $0.83(0.69$ to 0.97$)$ & $78 \%$ (49 to $95 \%$ ) & $85 \%$ (75 to $92 \%)$ & $46 \%$ (25 to $67 \%$ ) & $96 \%$ (88 to $99 \%$ ) \\
\hline & Pleural fluid & $\mathrm{n}=89$ & $0.86(0.78$ to 0.99$)$ & $92 \%$ (64 to $100 \%$ ) & $84 \%$ (74 to $92 \%$ ) & $50 \%$ (29 to $71 \%$ ) & $98 \%$ (92 to $100 \%$ ) \\
\hline \multirow{2}{*}{$\begin{array}{l}\text { Excluding } \\
\text { antibiotics for }>48 \mathrm{~h}\end{array}$} & Serum & $\mathrm{n}=81$ & $0.98(0.94$ to 1.00$)$ & $100 \%$ (48 to $100 \%$ ) & $86 \%$ (67 to $93 \%$ ) & $31 \%$ (11 to $59 \%)$ & $100 \%$ (95 to $100 \%$ ) \\
\hline & Pleural fluid & $\mathrm{n}=77$ & $0.89(0.79$ to 1.00$)$ & $80 \%$ (28 to $99 \%)$ & $81 \%(70$ to $89 \%)$ & $22 \%$ (6 to $48 \%$ ) & $98 \%$ (90 to $99 \%$ ) \\
\hline
\end{tabular}

there were no specimens or cultures were negative were analyzed in detail.

Results Most disease was pulmonary $(n=69)$. Other disease sites included lymph node $(\mathrm{n}=34)$, ocular $(\mathrm{n}=12)$ and pleura $(\mathrm{n}=10) .128$ (82\%) patients had samples sent for microbiology. 92 (59\%) patients were culture positive and $36(23 \%)$ were culture negative. 28 (18\%) patients had no specimens sent for culture. Factors which were associated with whether samples were sent for culture included site of disease $(p<0.0001)$, with ocular disease being the least likely to be sampled, and age of patients $(p=0.002) .45 \%$ patients $<17$ years did not have samples sent compared with $12.5 \%$ patients $17-64$. Ethnicity did not influence the frequency of sampling. A negative culture result was related to the specimen type $(p<0.0001)$ and patient's age $(p=0.019)$, with fewer paediatric samples positive. Site of disease or ethnicity did not affect culture results. In 25/28 cases with no microbiological specimens it was considered reasonable that specimens were not sent, as most of these were either ocular $(n=12)$ or paediatric ( $\mathrm{n}=9)$. In $3 / 28(11 \%)$ samples could have been sent and all involved adult patients not born in the UK who had procedures whereby specimens were sent only for histology. 32/36 culture negative cases were considered to have been managed appropriately. 4/36 (11\%) culture negative cases were potential missed opportunities for further sampling and were all due to patients with pleural TB not having pleural biopsies.

Conclusion In our centre, despite a microbiology negative rate of $41 \%$, reasonable opportunities to obtain a microbiological diagnosis are seldom missed.

\section{Investigating pleural disease \\ P63 A PROSPECTIVE OBSERVATIONAL TRIAL EXAMINING THE DIAGNOSTIC UTILITY OF SERUM AND PLEURAL FLUID PROCALCITONIN IN THE INITIAL INVESTIGATION OF UNILATERAL PLEURAL EFFUSIONS}

doi:10.1136/thx.2010.150979.14

${ }^{1} \mathrm{C}$ E Hooper, ${ }^{2} \mathrm{~A} J$ Morley, ${ }^{3} \mathrm{~J}$ E Harvey, ${ }^{1} \mathrm{~N}$ A Maskell. ${ }^{1}$ Academic Respiratory Unit, Department of Clinical Sciences, University of Bristol, Southmead Hospital, Bristol, UK; ${ }^{2}$ Pleural Clinical Trials Unit, Southmead Hospital, Bristol, UK; ${ }^{3}$ North Bristol Lung Centre, Southmead Hospital, Bristol, UK

Differentiation of pleural infection from other causes of pleural effusion, particularly pleural malignancy can be difficult. Clinical features and readily available tests are non-specific resulting in early over-diagnosis of pleural infection. Procalcitonin (PCT) is produced in response to acute bacterial infection and its measurement in serum has shown promise in directing and shortening antibiotic courses in lower respiratory tract infections. We prospectively examined the diagnostic accuracy of PCT in the investigation of unilateral pleural effusions.

Methods Consecutive patients with a unilateral pleural effusion, referred to a UK teaching hospital were included. Baseline serum and pleural fluid PCT was measured by enzyme-linked-fluorescent-assay (Vidas BRAHMS PCT-BioMerieux) and results compared to final diagnosis. Clinical data were collected prospectively and the ulti- mate diagnosis agreed against established criteria by two respiratory consultants, blind to the PCT result. Patients were followed up to histological or microbiological diagnosis, radiographic resolution or for 12 months.

Results 145 patients, median age 72 (31-96). 71 inpatients, 75 outpatients. Effusion diagnoses: Complicated parapneumonic (CPE) 26/145, Simple parapneumonic (SPE) 7/145, Malignant 73/145, Idiopathic pleuritis $4 / 145$, TB $1 / 145$, other benign cause $34 / 145$. Four patients with symptomatic non-thoracic bacterial infection and four with frank empyema were excluded from analysis. The Receiver operating characteristic (ROC) curve for serum PCT distinguishing CPE from non-infective diagnoses gave an AUC of 0.779 (95\% CI 0.658 to 0.899 ) and at an optimum cut-off of $0.09 \mathrm{ng} /$ $\mathrm{ml}$ had sensitivity $73 \%$, specificity $81 \%$, PPV $44 \%$ and NPV $94 \%$. The ROC curve for pleural PCT gave an AUC of 0.809 (95\% CI 0.709 to 0.908 ) and at an optimum cut-off of $0.1 \mathrm{ng} / \mathrm{ml}$ had sensitivity $81 \%$, specificity $78 \%$, PPV $45 \%$ and NPV 95\%. Sub-group analysis excluding patients with $>48 \mathrm{~h}$ in patient stay or $>48 \mathrm{~h}$ antibiotics further improved diagnostic accuracy. In patients with pleural fluid $\mathrm{pH} \leq 7.2$ serum PCT distinguished CPE from other effusions with an AUC of 0.808 (0.613-1.000).

Conclusion Serum and pleural fluid procalcitonin have promising diagnostic characteristics in distinguishing complicated parapneumonic effusions (Abstract P63 Table 1) from unilateral effusions of non-infective origin, having particularly high negative predictive values. Procalcitonin could help the clinician to decide optimal management pathways for individual patients.

\section{P64 COURSE AND VARIATION OF THE INTERCOSTAL ARTERY BY COMPUTED TOMOGRAPHY}

doi:10.1136/thx.2010.150979.15

${ }^{1} \mathrm{~N}$ M Rahman, ${ }^{2} \mathrm{E}$ J Helm, ${ }^{3} 0$ Talakoub, ${ }^{1} \mathrm{R}$ J 0 Davies, ${ }^{4} \mathrm{~F}$ V Gleeson. ${ }^{1}$ Oxford Centre for Respiratory Medicine, Oxford, UK; ${ }^{2}$ Department of Radiology, University Hospitals Coventry and Warwickshire NHS Trust, Coventry, UK; ${ }^{3}$ Department of Electrical and Computing Engineering, University of Toronto, Toronto, Canada; ${ }^{4}$ Department of Radiology, Oxford Radcliffe NHS Trust, Oxford, UK

Background The intercostal artery is thought to be shielded in the groove of the superior rib; however, the course and variability of the intercostal artery, and factors which may influence these, have not been described in vivo. Describing these variables in vivo has potentially important implications for avoiding complications during common pleural procedures.

Methods Maximum intensity projection (MIP) reformats in the coronal plane were produced from CT pulmonary angiograms, to identify the posterolateral course of the contrast opacified intercostal artery. A novel semi-automated computer segmentation algorithm was used to identify and measure distances between the lower border of the superior rib, the upper border of the inferior rib and the position of the intercostal artery when exposed in the intercostal space, and manually verified by a radiologist. Position and 
variability of the artery were described and then analysed for association with clinical factors using a random effects regression model. Results 298 arteries were analysed from 48 patients (mean age 60 years). The mean lateral distance from the spine over which the artery was exposed within the intercostal space ("unsafe artery length') was $39 \mathrm{~mm}$ with wide variability (SD=10 $\mathrm{mm}$, 10th to 90th centile $28 \mathrm{~mm}$ to $51 \mathrm{~mm}$, Abstract P64 Figure 1A). At $3 \mathrm{~cm}$ lateral distance from the spine $16.6 \%$ of arteries were shielded by the superior rib, compared to $96.6 \%$ at $6 \mathrm{~cm}$. Unsafe artery length was not associated with age, sex, rib space or side. Using regression modelling, variability of arterial position (as SD and coefficient of variation) was significantly associated with age (coeff $0.91, \mathrm{p}<0.001)$ and rib space number (coeff $-2.60, \mathrm{p}<0.001$ ) (Abstract P64 Figure 1B). Variability of arterial position was strongly negatively correlated with lateral distance from the spine (Pearson's $-0.77, \mathrm{p}<0.001$ ).
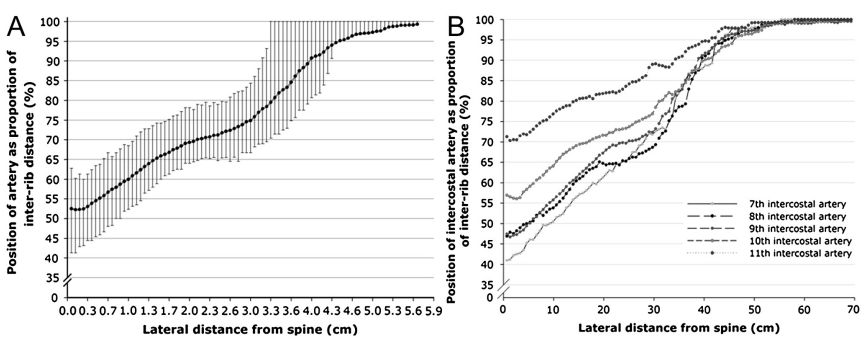

Abstract P64 Figure 1 (A). Position of artery as proportion of inter-rib distance (\%). (B). Position of intercostal artery as proportion of inter-rib distance (\%).

Conclusions The intercostal artery is exposed within the intercostal space in the first $6 \mathrm{~cm}$ lateral to the spine; variability of its vertical position is greater in older patients and more cephalad rib spaces. This implies that pleural interventions within $6 \mathrm{~cm}$ lateral to the spine should be conducted with caution and that the risk of intercostal artery laceration is potentially higher in older patients and more cephalad rib spaces.

\section{P65 IS SERUM N-TERMINAL PRO B TYPE NATRIURETIC PEPTIDE (NT-PROBNP) MEASUREMENT USEFUL IN THE INVESTIGATION OF UNILATERAL PLEURAL EFFUSIONS?-A PROSPECTIVE OBSERVATIONAL STUDY}

doi:10.1136/thx.2010.150979.16

${ }^{1} \mathrm{C}$ E Hooper, ${ }^{2} \mathrm{l}$ Rider, ${ }^{3} \mathrm{R}$ S Finn, ${ }^{4} \mathrm{~A} J \mathrm{~J}$ Morley, ${ }^{3} \mathrm{~J}$ E Harvey, ${ }^{2} \mathrm{~A}$ Skyrme-Jones, ${ }^{1} \mathrm{~N}$ A Maskell. ${ }^{1}$ Academic Respiratory Unit, Department of Clinical sciences, University of Bristol, Southmead Hospital, Bristol, UK; ${ }^{2}$ Southmead Hospital, North Bristol NHS Trust, Bristol, UK; ${ }^{3}$ North Bristol Lung Centre, Southmead Hospital, Bristol, UK; ${ }^{4}$ Pleural Clinical Trials Unit, Southmead Hospital, Bristol, UK

Measurement of serum NT-proBNP has been proposed in the investigation of pleural effusions, particularly in the diagnosis of cardiac failure in those misclassified as exudates by Light's criteria. Studies have reported excellent diagnostic accuracy for the test but have included both bilateral and unilateral effusions and applied short follow-up periods. We prospectively examined the diagnostic utility of serum NT-proBNP in a consecutive series of unilateral pleural effusions with robust follow-up and diagnostic criteria.

Method Consecutive patients presenting to a UK teaching hospital with an undiagnosed unilateral pleural effusion underwent clinical assessment including CXR, ECG, echocardiogram, thoracentesis (and CT when appropriate). Light's criteria were applied. Serum NTproBNP was measured using point of care ELISA. Patients were followed up to histological/microbiological diagnosis, radiographic resolution or 12 months. Echocardiograms were double reported and diagnosis determined independently by two respiratory consultants-all blind to NT-proBNP results.

Results 118 patients. Median age 74 (42-95). 39 in patients, 79 outpatients. 18 transudates, 92 exudates. 30 large, 66 moderate, 22 small. Diagnoses: primary cardiac cause (PCC) 20/118, Malignant $57 / 118$, PE $4 / 118$, Non-cardiac transudate $3 / 118$, other benign cause 30/118. The ROC curve for NT-proBNP discriminating effusions of PCC gave an AUC of 0.845 (0.774-0.934). At cut-off of age and sex adjusted 97.5 th centile (healthy population) NT-proBNP had sensitivity $100 \%$, Specificity 53\%, PPV 30\% and NPV 100\% and all four cardiac exudates were correctly diagnosed. At an optimum cutoff of $1500 \mathrm{pg} / \mathrm{ml}$-sensitivity $75 \%$, specificity $76 \%$, PPV $38 \%$ and NPV 94\%. Co-morbid cardiac disease was common in patients without a PCC for effusion with $70 \%$ having significant abnormalities on echocardiogram but cardiac disease was considered to be contributing to effusion in only $9 / 98$ of this group.

Conclusion The excellent negative predictive value of NT-proBNP, particularly at an age and sex adjusted cut-off level gives the test utility to rule out a primarily cardiac cause in selected cases of unilateral pleural effusion. Co-morbid cardiac disease and associated NT-proBNP elevation is very common in patients with a noncardiac origin of pleural effusion such that a positive test at baseline should not alter the initial investigation pathway, particularly amongst pleural exudates.

\section{P66 HISTONE DEACETYLASE INHIBITOR CBHA ATTENUATES THE EXPRESSION OF PLASMINOGEN ACTIVATOR INHIBITOR-1 IN HUMAN PLEURAL MESOTHELIAL CELLS}

doi:10.1136/thx.2010.150979.17

${ }^{1}$ Chi-Li Chung, ${ }^{2}$ Wei-Lin Chen, ${ }^{3} Y u-W e n$ Cheng, ${ }^{2}$ Yung-Chen Chou, ${ }^{2}$ Ming-Jen Hsu, ${ }^{1}$ Che-Jen Hsiao, ${ }^{2}$ Joen-Rong Sheu, ${ }^{2}$ George Hsiao. ${ }^{1}$ Department of Chest Medicine, Taipei Medical University Hospital and School of Respiratory Therapy, College of Medicine, Taipei Medical University, Taipei, Taiwan; ${ }^{2}$ Graduate Institute of Medical Sciences and Department of Pharmacology, College of Medicine, Taipei Medical University, Taipei, Taiwan; ${ }^{3}$ Department of Pharmacy, School of Pharmacy, Taipei Medical University, Taipei, Taiwan

Background Plasminogen activator inhibitor-1 (PAI-1), primarily upregulated by transforming growth factor (TGF)- $\beta$, is essential for development of fibrosis. Histone deacetylases (HDACs) have been shown to modulate gene expression and fibrogenesis in various tissues. However, the implications of HDAC in PAI-1 expression and pleural fibrosis remain unclear. We examined the effects of $m$-carboxycinnamic acid bis-hydroxamide (CBHA), a hybrid-polar HDAC inhibitor, on PAI-1 expression in a human pleural mesothelial cell line (MeT-5A). Methods MeT-5A cells were treated with TGF- $\beta 1(10 \mathrm{ng} / \mathrm{ml})$ in the presence or absence of CBHA $(0.2-1 \mu \mathrm{M})$. The expression and stability of PAI-1 mRNA and protein, PAI-1 promoter activity, activation of Smad signaling, and protein-protein interactions of Smads with transcriptional cofactors Sp1 and coactivator p300 were assayed using the methods of Western blotting, reverse transcription-polymerase chain reaction, transient transfection and luciferase activity assay, immunoflurescence staining and immunoprecipitation, respectively.

Results CBHA significantly inhibited TGF- $\beta 1$-induced PAI- 1 mRNA and protein expression, and attenuated PAI-1 promoter activity in MeT-5A cells. CBHA abrogated TGF- $\beta 1$-induced Smad4 nuclear translocation, but not Smad2/3 activation. Furthermore, the TGF$\beta 1$-induced association of Smad4 with p300, but not with Sp1, was disrupted by CBHA. Alternatively, CBHA accelerated PAI-1 mRNA degradation, possibly through suppression of the mRNA stabilizing protein nucleolin (Abstract P66 Figure 1). 